BMJ Open Diabetes Research \& Care

\title{
Biomarkers of tubulointerstitial damage and function in type 1 diabetes
}

Ian $\mathrm{H}$ de Boer, ${ }^{1}$ Xiaoyu Gao, ${ }^{2}$ Ionut Bebu, ${ }^{2}$ Andrew $\mathrm{N}$ Hoofnagle, ${ }^{3}$ John M Lachin, ${ }^{2}$ Andrew Paterson, ${ }^{4}$ Bruce A Perkins, ${ }^{5}$ Amy K Saenger, ${ }^{6}$ Michael W Steffes, ${ }^{6}$ Bernard Zinman, ${ }^{7}$ Mark E Molitch, ${ }^{8}$ Diabetes Control and Complications Trial/ Epidemiology of Diabetes Interventions and Complications (DCCT/EDIC) Research Group.

To cite: de Boer IH, Gao X, Bebu I, et al. Biomarkers of tubulointerstitial damage and function in type 1 diabetes. BMJ Open Diab Res Care 2017;5:e000461. doi:10.1136/ bmjdrc-2017-000461

Received 10 August 2017 Revised 9 October 2017 Accepted 27 October 2017

\section{(a) CrossMark}

${ }^{1}$ Division of Nephrology and Kidney Research Institute, University of Washington, Seattle, Washington, USA ${ }^{2}$ The George Washington University, Rockville, Maryland, USA

${ }^{3}$ Department of Laboratory Medicine and Kidney Research Institute, University of Washington, Seattle, Washington, USA

${ }^{4}$ Hospital for Sick Children, University of Toronto, Toronto, Ontario, Canada

${ }^{5}$ Division of Endocrinology and Metabolism, University of Toronto and the Lunenfeld Tanenbaum Research Institute, Mount Sinai Hospital, University of Toronto, Toronto, Ontario, Canada

${ }^{6}$ University of Minnesota, Minnesota, Minneapolis, USA ${ }^{7}$ Lunenfeld Tanenbaum Research Institute, Mount Sinai Hospital, University of Toronto, Toronto, Ontario, Canada ${ }^{8}$ Northwestern University, Chicago, Illinois, USA

Correspondence to Dr lan H de Boer; deboer@u.washington.edu

\section{ABSTRACT}

Objective To evaluate biomarkers of renal tubulointerstitial damage and function in type 1 diabetes with and without diabetic kidney disease.

Research design and methods Cross-sectional casecontrol study of Diabetes Control and Complications Trial/ Epidemiology of Diabetes Interventions and Complications Study participants. Cases $(\mathrm{N}=43)$ had incident persistent estimated glomerular filtration rate (eGFR) $<60 \mathrm{~mL} /$ $\mathrm{min} / 1.73 \mathrm{~m}^{2}$ with urinary albumin excretion $\geq 300 \mathrm{mg} / 24$ hour. Controls $(\mathrm{N}=43)$ had persistent eGFR $\geq 90 \mathrm{~mL} /$ $\min / 1.73 \mathrm{~m}^{2}$ and urinary albumin excretion $<30 \mathrm{mg} / 24$ hour. Urinary and plasma biomarkers reflecting tubular injury, inflammation, fibrosis, secretion, and synthetic function were measured from stored specimens collected at the first study visit with reduced eGFR (for case participants) or the corresponding study year (for control participants).

Results Mean (SD) age was 51 (9) and 50 (8) years for case and control participants, and mean (SD) duration of diabetes was 30 (6) and 30 (5) years, respectively. Mean (SD) eGFR was 39 (14) and 103 (9) $\mathrm{mL} / \mathrm{min} / 1.73 \mathrm{~m}^{2}$ for case and control participants, and mean (SD) albumin excretion rate was 1978 (2914) and 10 (7) mg/day, respectively. Comparing cases with controls, significant differences were observed in each measured biomarker, including urine epidermal growth factor (mean 5.3 vs $21.2 \mu \mathrm{g} / \mathrm{g}$ creatinine for case vs control participants, respectively), urine monocyte chemoattractant protein-1 (596 vs $123 \mathrm{ng} / \mathrm{g}$ creatinine), urine galectin-3 (168 vs 52 $\mu \mathrm{g} / \mathrm{g}$ creatinine), plasma soluble tubular necrosis factor receptor-1 (3695 vs 1022 pg/mL), plasma galectin-3 (21.3 vs $11.0 \mathrm{ng} / \mathrm{mL}$ ), urinary clearances of hippurate (70 vs 167 $\mathrm{mL} / \mathrm{min}$ ) and cinnamoylglycine (77 vs $317 \mathrm{~mL} / \mathrm{min})$, and plasma arginine-citrulline ratio (5.6 vs $7.7 \mu \mathrm{g} / \mu \mathrm{g})$, each $\mathrm{P}<0.001$.

Conclusions Marked abnormalities in biomarkers of kidney tubular injury, inflammation, fibrosis, secretion, and synthetic function accompany reduced eGFR and albuminuria in type 1 diabetes.

Trial registration number NCT00360893, NCT00360815.

\section{INTRODUCTION}

Elevated urine albumin excretion (albuminuria) and reduced estimated glomerular filtration rate (eGFR) have long been viewed as

\section{Significance of this study}

What is already known about this subject? Kidney disease is a common complication of types 1 and 2 diabetes. Diabetic kidney disease is usually assessed using estimated glomerular filtration rate (eGFR) and urine albumin excretion, which predominantly reflect damage to the kidney glomerulus.

\section{What are the new findings?}

Compared with people with type 1 diabetes and normal eGFR and urine albumin excretion, those with abnormal eGFR and urine albumin excretion had significant concomitant abnormalities in urine and plasma biomarkers that reflect damage and impaired function of the tubulointerstitital compartment of the kidney. The results suggest that tubular injury, inflammation, and fibrosis as well as impairments in kidney tubule secretion and synthesis of small molecules are prominent features of diabetic kidney disease.

How might these results change the focus of research or clinical practice?

This study suggests that diverse aspects of tubulointerstitial damage and function should be evaluated in future studies of diabetic kidney disease and provides new information on specific biomarkers that might be useful for this work. Ultimately, such studies may better define the pathophysiology of diabetic kidney disease, help develop new biomarkers to add to eGFR and urine albumin excretion in clinical care, and guide the development of new treatments for diabetic kidney disease.

the cardinal manifestations of diabetic kidney disease (DKD). These DKD manifestations are common in type 1 diabetes, can progress to end-stage renal disease, and are strongly associated with cardiovascular diseases and other adverse health outcomes. ${ }^{1}$ However, kidney functions beyond glomerular filtration and conservation of circulating proteins are also critical for maintaining homeostasis and health. In particular, renal tubular cells secrete small molecules and synthesize growth 
factors, hormones, and amino acids. Renal tubulointerestitial damage - not glomerular disease-is most strongly associated with progression of chronic kidney disease. ${ }^{2,3}$ Further understanding of tubular damage, secretion, and synthesis may lead to better understanding of kidney injury in $\mathrm{DKD}$, the relationship of kidney disease to other diabetes complications, and new therapeutic targets.

\section{Research design and methods}

We performed a cross-sectional case-control study nested within the Diabetes Control and Complications Trial/ Epidemiology of Diabetes Interventions and Complications (DCCT/EDIC) Study. The goal of the study was to identify candidate biomarkers of tubulointerstitial damage and function that are markedly abnormal in $\mathrm{DKD}$ in order to prioritize promising biomarkers for additional longitudinal studies.

We studied all participants with incident persistent eGFR $<60 \mathrm{~mL} / \mathrm{min} / 1.73 \mathrm{~m}^{2}$ (on two consecutive study visits), urinary albumin excretion rate (AER) $\geq 300 \mathrm{mg} / 24$ hour, and available biosamples $(\mathrm{N}=43)$. Controls $(\mathrm{N}=43)$ were randomly selected from DCCT/EDIC Study participants who maintained persistent eGFR $\geq 90 \mathrm{~mL} / \mathrm{min} / 1.73 \mathrm{~m}^{2}$ and AER $<30 \mathrm{mg} / 24$ hour through the study visit on which the corresponding case participant developed incident eGFR $<60 \mathrm{~mL} / \mathrm{min} / 1.73 \mathrm{~m}^{2}$. Control participants were matched to cases on duration of diabetes and DCCT cohort (primary vs secondary intervention).

Using stored urine and plasma specimens collected at the time of incident persistent eGFR $<60 \mathrm{~mL} /$ $\min / 1.73 \mathrm{~m}^{2}$ (for cases) or at the same study year (for controls), we measured biomarkers reflecting tubular injury, inflammation, fibrosis, secretion, and synthetic function. These included urine epidermal growth factor (EGF), urine monocyte chemoattractant protein-1 (MCP-1), plasma soluble tubular necrosis factor receptor-1 (sTNFR-1), urine and plasma galectin-3, the urinary clearances of hippurate and cinnamoylglycine, and plasma arginine-citrulline ratio. These biomarkers were chosen to represent diverse aspects of tubulointerstitial damage and function that are not captured well by eGFR and albuminuria, prioritizing those most likely to be feasible and useful based on published literature. ${ }^{4-9}$

Aliquots of plasma and urine were collected concurrently using standardized procedures and stored at $<-70^{\circ} \mathrm{C}$. For 66 participants (with samples collected prior to August 2012), urine aliquots were taken from 4-hour collections. For 20 participants (with samples collected after August 2012), urine was taken from a random morning sample, based on a change in the EDIC Study protocol. Urine EGF, MCP-1, and sTNFR-1 were measured using ELISA assays from R\&D Systems. Urine and plasma galectin-3 were measured using ELISA assays from BGMedicine. Plasma and urine hippurate and cinnamoylglycine and plasma arginine and citrulline were measured by mass spectrometry. 8,10

Urinary EGF, MCP-1, and sTNFR-1 were standardized to urine creatinine concentration. The urinary clearances of hippurate and cinnamoylglycine were calculated within the subset of 66 participants with timed urine collections as urinary excretion rate divided by plasma concentration. Differences in biomarkers were tested using the paired T-test, based on the case-control design.

\section{RESULTS}

Mean age was 50.5 years and mean duration of diabetes was 30.1 years at the time of biospecimen collection. Case and control participants were similar with regard to characteristics used for matching (DCCT cohort and duration of diabetes) as well as age and gender (table 1). Mean (SD) eGFR was 39 (14) and 103 (9) mL/ $\mathrm{min} / 1.73 \mathrm{~m}^{2}$ for case and control participants, and mean (SD) albumin excretion rate was 1978 (2914) and 10 (7) $\mathrm{mg}$ /day, respectively. Blood pressure and hemoglobin A1c (HbA1c) (particularly time-weighted HbA1c) were higher comparing case with control participants.

The distributions of each measured biomarker differed significantly comparing cases with controls (table 2 ). The urinary excretion of EGF was markedly lower in case versus control participants, while the urinary excretion of MCP-1 and galectin-3 were markedly higher. Plasma sTNFR-1 and galectin-3 concentrations were markedly higher in case versus control participants, while plasma arginine-citrulline ratio (a marker of tubular synthetic function) was lower. The urinary clearances of hippurate and cinnamoylglycine were significantly lower in case versus control participants. For urinary biomarkers, results were similar among subsets of participants with timed compared with random urine samples.

\section{DISCUSSION}

We observed marked abnormalities in biomarkers of tubulointerstitial damage and function comparing participants with type 1 diabetes, incident eGFR $<60 \mathrm{~mL}$ / $\mathrm{min} / 1.73 \mathrm{~m}^{2}$, and AER $\geq 300 \mathrm{mg} / 24$ hour to those without evidence of kidney disease. Differences in all eight candidate biomarkers were large and statistically significant, despite the modest sample size. The eight biomarkers represent diverse aspects of tubulointerstitial damage and function, including tubular injury, inflammation, fibrosis, secretion, and synthetic function. Our results demonstrate that $\mathrm{DKD}$ in type 1 diabetes is characterized by a broad range of abnormalities beyond reduced eGFR and albuminuria, and suggest that diverse facets of tubulointerstitial damage and function should be evaluated in future studies.

Tubular damage, inflammation, and tubulointerstitial fibrosis are acknowledged mechanisms of DKD. 2,6,11-13 Therefore, the observation that markers of these processes are abnormal among patients with established DKD is not surprising. However, the large differences we observed reinforce the concept that tubulointerstitial damage is a critical feature of DKD. Moreover, markers of tubular injury, inflammation, fibrosis, secretion, and 
Table 1 Clinical characteristics of included DCCT/EDIC Study participants with and without kidney disease

\begin{tabular}{llc|}
\hline & \multicolumn{2}{l}{$\begin{array}{l}\text { Kidney disease } \\
\text { status* }\end{array}$} \\
\cline { 2 - 3 } & $\begin{array}{l}\text { Controls } \\
\text { (N=43) }\end{array}$ & $\begin{array}{l}\text { Cases } \\
\text { (N=43) }\end{array}$ \\
\hline Clinical characteristics & & \\
\hline Demographic data & & \\
\hline Age (years) & $50(8)$ & $51(9)$ \\
\hline Duration of diabetes (years) & $30(5)$ & $30(6)$ \\
\hline Female gender & $20(47)$ & $17(40)$ \\
\hline DCCT intensive therapy & $21(49)$ & $14(33)$ \\
\hline DCCT primary cohort & $22(51)$ & $22(51)$ \\
\hline Physical examination data & & \\
\hline Body mass index (kg/m²) & $29.3(5.2)$ & $30.4(6.0)$ \\
\hline Systolic BP (mmHg) & $122(14)$ & $137(21)$ \\
\hline Diastolic BP (mmHg) & $75(9)$ & $76(13)$ \\
\hline Laboratory data & & \\
\hline eGFR (mL/min/1.73 m²) & $103(9)$ & $39(14)$ \\
\hline Albumin excretion rate (mg/day) & $10(7)$ & $1978(2914)$ \\
\hline Current HbA1c (\%) & $8.0(1.0)$ & $8.6(1.7)$ \\
\hline DCCT/EDIC time-weighted & $7.9(0.6)$ & $9.2(1.1)$ \\
\hline HbA1c (\%) & & \\
\hline EDIC time-weighted HbA1c (\%) & $7.9(0.7)$ & $9.1(1.3)$ \\
\hline LDL cholesterol (mg/dL) & $95(29)$ & $105(39)$ \\
\hline HDL cholesterol (mg/dL) & $60(19)$ & $52(18)$ \\
\hline Triglycerides (mg/dL) & $77(35)$ & $131(73)$ \\
\hline
\end{tabular}

Cell contents are mean (SD) or N (\%).

${ }^{*}$ Cases were defined by incident persistent eGFR $<60 \mathrm{~mL}$ $\mathrm{min} / 1.73 \mathrm{~m}^{2}$ with urinary AER $\geq 300 \mathrm{mg} / 24$ hour; control subjects were randomly selected from the pool of DCCT/EDIC Study participants who maintained persistent eGFR $\geq 90 \mathrm{~mL} / \mathrm{min} / 1.73$ $\mathrm{m}^{2}$ and AER $<30 \mathrm{mg} / 24$ hour through the study visit on which the corresponding case participant developed incident eGFR $<60 \mathrm{~mL} /$ $\mathrm{min} / 1.73 \mathrm{~m}^{2}$ and were additionally matched to cases on duration of diabetes and DCCT cohort.

AER, albumin excretion rate; BP, blood pressure; DCCT/EDIC, Diabetes Control and Complications Trial/Epidemiology of Diabetes Interventions and Complications; eGFR, estimated glomerular filtration rate; HDL, high-density lipoprotein; $\mathrm{HbA1c}$, hemoglobin A1c; LDL. low-density lipoprotein.

synthetic function are not currently used in clinical care and may ultimately be useful adjuncts to eGFR and albuminuria. Our data identify promising biomarkers to evaluate in future longitudinal studies that evaluate this possibility.

Recently, in a diverse population of people with chronic kidney disease (most without diabetes), an agnostic analysis starting with transcriptomics of kidney biopsy tissue ultimately identified reduced urinary EGF as a strong predictor of progressive kidney disease. ${ }^{4}$ EGF is a growth factor produced by distal tubular cells that promotes tubular cell repair and regeneration. Membrane-bound TNFR-1 is involved in apoptosis, survival, and key aspects
Table 2 Biomarkers of tubulointerstitial damage and function among included DCCT/EDIC Study participants with and without kidney disease

\begin{tabular}{|c|c|c|c|}
\hline & \multicolumn{2}{|c|}{ Kidney disease status* } & \multirow[b]{2}{*}{$\begin{array}{l}P \text { value for } \\
\text { difference }\end{array}$} \\
\hline & $\begin{array}{l}\text { Controls } \\
(\mathrm{N}=43)\end{array}$ & $\begin{array}{l}\text { Cases } \\
(\mathrm{N}=43)\end{array}$ & \\
\hline \multicolumn{4}{|l|}{ Urine biomarkers $†$} \\
\hline EGF $(\mu \mathrm{g} / \mathrm{g})$ & $21.2(8.7)$ & $5.3(2.8)$ & $<0.0001$ \\
\hline MCP-1 (ng/g) & $123(100)$ & $596(860)$ & $<0.0001$ \\
\hline Galectin-3 ( $\mu \mathrm{g} / \mathrm{g})$ & $52(35)$ & $168(145)$ & $<0.0001$ \\
\hline \multicolumn{4}{|l|}{ Plasma biomarkers } \\
\hline sTNFR-1 (pg/mL) & $1022(256)$ & 3695 (1289) & $<0.0001$ \\
\hline Galectin-3 (ng/mL) & $11.0(5.3)$ & $21.3(6.6)$ & $<0.0001$ \\
\hline $\begin{array}{l}\text { Arginine-citrulline } \\
\text { ratio }(\mu \mathrm{g} / \mu \mathrm{g})\end{array}$ & $7.7(2.8)$ & $5.6(1.9)$ & 0.0004 \\
\hline \multicolumn{4}{|l|}{ Urinary clearance } \\
\hline Hippurate (mL/min) & $167(72)$ & $70(79)$ & $<0.0001$ \\
\hline $\begin{array}{l}\text { Cinnamoylglycine } \\
\text { (mL/min) }\end{array}$ & 317 (150) & $77(71)$ & $<0.0001$ \\
\hline
\end{tabular}

Cell contents are mean (SD).

${ }^{*}$ Cases were defined by incident persistent eGFR $<60 \mathrm{~mL}$ $\mathrm{min} / 1.73 \mathrm{~m}^{2}$ with urinary AER $\geq 300 \mathrm{mg} / 24$ hour; control subjects were randomly selected from the pool of DCCT/EDIC Study participants who maintained persistent eGFR $\geq 90 \mathrm{~mL} / \mathrm{min} / 1.73$ $\mathrm{m}^{2}$ and $A E R<30 \mathrm{mg} / 24$ hour through the study visit on which the corresponding case participant developed incident eGFR $<60 \mathrm{~mL} /$ $\mathrm{min} / 1.73 \mathrm{~m}^{2}$ and were additionally matched to cases on duration of diabetes and DCCT cohort.

†Urinary biomarkers are expressed per gram of urine creatinine. fUrinary clearances are restricted to 66 participants with timed urine collections.

AER, albumin excretion rate; DCCT/EDIC, Diabetes Control and Complications Trial/Epidemiology of Diabetes Interventions and Complications; eGFR, estimated glomerular filtration rate; MCP-1, monocyte chemoattractant protein-1; sTNFR-1, plasma soluble tubular necrosis factor receptor-1.

of inflammation and immune response. The soluble form, sTNFR-1, was previously associated with rapid kidney function decline and incident eGFR $<60 \mathrm{~mL} / \mathrm{min} / 1.73 \mathrm{~m}^{2}$ in the Joslin study of type 1 diabetes. ${ }^{5}$ Urine MCP-1, a more specific marker of renal inflammation, was associated with eGFR loss in type 2 diabetes. ${ }^{6}$ Plasma concentration of GDF-15, a member of the TGF- $\beta$ cytokine superfamily, correlated with GDF-15 expression in renal tubular cells and was associated with progression of kidney disease in populations with established chronic kidney disease (with and without diabetes). ${ }^{7}$

Tubular functions, including secretion and synthetic function, have been less comprehensively studied in DKD. Many proposed uremic toxins are small organic molecules that circulate bound to plasma proteins, such as albumin, and are poorly filtered but actively secreted by proximal tubular cells. Hippurate and cinnamoylglycine are examples of such small molecules, which accumulate with kidney disease in a manner that is not necessarily proportional to reduction in GFR. ${ }^{8}$ Renal tubular cells also perform many synthetic functions, such as the production of arginine from citrulline. $^{9}$ Additional renal synthetic functions include 
production of hydroxylated vitamin D metabolites, gluconeogenesis, ammoniagenesis, and erythropoietin production. Accumulation of uremic solutes and reduced synthesis of important nutrients and hormones may promote insulin resistance, atherosclerosis, and other pathologic processes contributing to adverse health outcomes of chronic kidney disease. $^{8,14,15}$ Our results suggest that biomarkers of tubulointerstitial damage and function previously examined in other populations and evaluated in this study may be useful in type 1 diabetes.

The main limitation of our study is the cross-sectional design, from which we cannot determine the sequence of change in renal biomarkers or the utility of tubulointerstitial biomarkers for predicting kidney disease progression or other complications. Compared with control participants, those with DKD had higher systolic blood pressure and a history of worse glycemic control, but we cannot determine the extent to which hyperglycemia and hypertension contributed to tubulointerstitial damage and impaired tubular function. We are unable to discern whether urine biomarkers arise from the tubulointerstitial compartment of the kidney or appear in the urine through filtration. In addition, our candidate biomarker approach necessarily excludes some potentially important biomarkers. Study strengths include the simultaneous evaluation of biomarkers reflecting multiple aspects of tubulointersititial damage and function, the well-characterized cohort with robust control subjects, the use of novel and precise mass spectrometry assays for some analytes, and the strength of the observed associations.

\section{CONCLUSION}

In type 1 diabetes, marked abnormalities in biomarkers of kidney tubular injury, inflammation, fibrosis, secretion, and synthetic function accompany reduced eGFR and albuminuria. Longitudinal studies are needed to determine the time course over which these biomarkers of tubulointerstitial function change relative to eGFR and albuminuria and to determine whether these biomarkers are associated with DKD progression and complications. Biomarkers of tubulointerstitial damage and function may be useful for the development of new therapies targeting DKD.

\section{Acknowledgements A complete list of participants in the DCCT/EDIC Research Group can be found in the New England Journal of Medicine, 2011;365:2366- 2376.}

Contributors All authors contributed to the study design, interpreted the data, revised the manuscript critically for important intellectual content, approved the final version of the manuscript and agreed to be accountable for the work. In addition, IHdeB drafted the manuscript; XG and IB and JML performed analyses and $\mathrm{ANH}, \mathrm{AS}$ and MWS acquired laboratory data.

Funding The DCCT/EDIC has been supported by U01 Cooperative Agreement grants (1982-93, 2011-2016), and contracts (1982-2011) with the Division of Diabetes Endocrinology and Metabolic Diseases of the National Institute of Diabetes and Digestive and Kidney Disease (NIDDK), and through support by the National Eye Institute, the National Institute of Neurologic Disorders and Stroke, the Genetic Clinical Research Centers Program (1993- 2007) and Clinical Translational Science Center Program (2006-present), Bethesda, Maryland, USA. Industry contributors have had no role in the DCCT/EDIC study, but have provided free or discounted supplies or equipment to support participants' adherence to the study:
Abbott Diabetes Care (Alameda, CA), Animas (Westchester, PA), Bayer Diabetes Care (North America Headquarters, Tarrytown, NY) Becton Dickinson (Franklin Lakes, NJ), CanAm (Atlanta, GA), Eli Lilly (Indianapolis, IN), Lifescan (Milpitas, CA), Medtronic Diabetes (Minneapolis, MN), Omron (Shelton, CT), OmniPod Insulin Management System (Bedford, MA), Roche Diabetes Care (Indianapolis, IN) and Sanofi-Aventis (Bridgewater, NJ). Dr de Boer's effort was supported by grants R01DK087726 and R01DK088762 from the NIDDK.

Competing interests IHdeB consulted for Boehringer-Ingelheim and Ironwood and received research equipment and supplies from Medtronic and Abbott. BAP has consulted for Neurometrix, Boehringer-Ingelheim, Abbott and Insulet. He has received speaker fees for medical education events from Medtronic, Novo Nordisk, Abbott, Insulet and Janssen. His institution has received support on his behalf for research funded by Boehringer-Ingelheim and Novo Nordisk.

Patient consent Obtained.

Ethics approval Site IRBs.

Provenance and peer review Not commissioned; externally peer reviewed. Data sharing statement Data from the DCCT/EDIC cohort are available to the public through the NIDDK Repository.

Open Access This is an Open Access article distributed in accordance with the Creative Commons Attribution Non Commercial (CC BY-NC 4.0) license, which permits others to distribute, remix, adapt, build upon this work non-commercially, and license their derivative works on different terms, provided the original work is properly cited and the use is non-commercial. See: http://creativecommons.org/ licenses/by-nc/4.0/

(C) Article author(s) (or their employer(s) unless otherwise stated in the text of the article) 2017. All rights reserved. No commercial use is permitted unless otherwise expressly granted.

\section{REFERENCES}

1. de Boer IH. DCCT/EDIC Research Group. Kidney disease and related findings in the diabetes control and complications trial/ epidemiology of diabetes interventions and complications study. Diabetes Care 2014:37:24-30.

2. Nath KA. Tubulointerstitial changes as a major determinant in the progression of renal damage. Am J Kidney Dis 1992;20:1-17.

3. Mise K, Hoshino J, Ueno T, et al. Prognostic value of tubulointerstitial lesions, urinary N-Acetyl- $\beta$-d-glucosaminidase, and urinary $\beta 2-$ microglobulin in patients with type 2 diabetes and biopsy-proven diabetic nephropathy. Clin J Am Soc Nephrol 2016;11:593-601.

4. Ju W, Nair V, Smith S, et al. Tissue transcriptome-driven identification of epidermal growth factor as a chronic kidney disease biomarker. Sci Transl Med 2015;7:316ra193.

5. Gohda T, Niewczas MA, Ficociello LH, et al. Circulating TNF receptors 1 and 2 predict stage 3 CKD in type 1 diabetes. J Am Soc Nephrol 2012;23:516-24.

6. Nadkarni GN, Rao V, Ismail-Beigi F, et al. Association of urinary biomarkers of inflammation, Injury, and fibrosis with renal function decline: the ACCORD trial. Clin J Am Soc Nephrol 2016;11:1343-52.

7. Nair V, Robinson-Cohen C, Smith MR, et al. Growth differentiation factor-15 and risk of CKD progression. J Am Soc Nephrol 2017;28:2233-40.

8. Suchy-Dicey AM, Laha T, Hoofnagle A, et al. Tubular secretion in CKD. J Am Soc Nephrol 2016;27:2148-55.

9. Rhee EP, Clish CB, Ghorbani A, et al. A combined epidemiologic and metabolomic approach improves CKD prediction. J Am Soc Nephrol 2013;24:1330-8.

10. Brown CM, Becker JO, Wise PM, et al. Simultaneous determination of $6 \mathrm{~L}$-arginine metabolites in human and mouse plasma by using hydrophilic-interaction chromatography and electrospray tandem mass spectrometry. Clin Chem 2011;57:701-9.

11. Breyer MD, Susztak K. The next generation of therapeutics for chronic kidney disease. Nat Rev Drug Discov 2016;15:568-88.

12. Gilbert RE. Proximal tubulopathy: prime mover and key therapeutic target in diabetic kidney disease. Diabetes 2017;66:791-800.

13. Bader $\mathrm{R}$, Bader $\mathrm{H}$, Grund $\mathrm{KE}$, et al. Structure and function of the kidney in diabetic glomerulosclerosis. Correlations between morphological and functional parameters. Pathol Res Pract 1980:167(2-4):204-16.

14. Koppe L, Pillon NJ, Vella RE, et al. p-Cresyl sulfate promotes insulin resistance associated with CKD. J Am Soc Nephrol 2013;24:88-99.

15. Wang Z, Klipfell E, Bennett BJ, et al. Gut flora metabolism of phosphatidylcholine promotes cardiovascular disease. Nature 2011:472:57-63. 